K.A. van der Hucht, G. Koenigsberger \& P.R.J. Eenens, eds.

\title{
A search for new stellar wind sources using the Canadian Galactic Plane Survey (CGPS)
}

\author{
Serge Pineault \\ Département de physique, Université Laval, Québec, Canada
}

\begin{abstract}
The CGPS is a project undertaken at the Dominion Radio Astrophysical Observatory $(D R A O)$, in collaboration with a consortium of university astronomers, to image the atomic hydrogen and the radio continuum emission from the interstellar medium of the Galaxy. We describe the contributions this project can make to WR star research, in particular, and to the study of stellar wind sources, in general. Specific examples are presented.
\end{abstract}

\section{Results}

The CGPS stands for a systematic radio mapping of the Galaxy in the region $75^{\circ}<l<145^{\circ}$ and $-3^{\circ}<b<+5^{\circ}$. A more detailed description can be found at $<$ http://www.ras.ucalgary.ca/GPS_pub.html $>$ and results of the pilot survey in Normandeau et al. (1996). The CGPS makes it possible to survey the position of all currently known WR stars as well as of other stars suspected of possessing a significant stellar wind (SW). It also provides a homogeneous data-base from which to search for new structures possessing a partial or complete shell morphology suggestive of SW action. Discrimination with other similarly looking
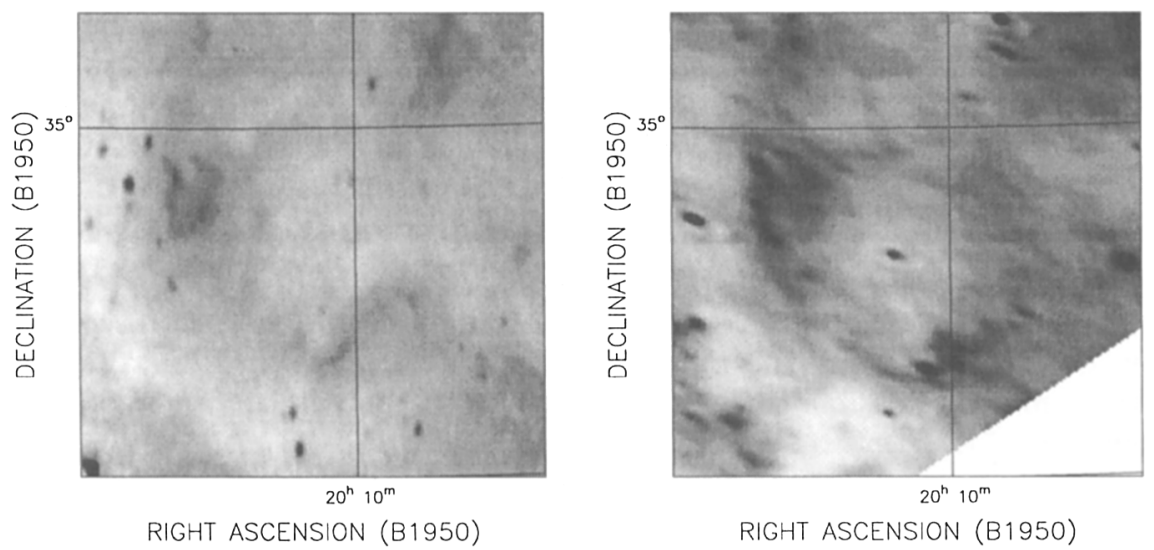

RIGHT ASCENSION (B1950)

Figure 1. A new SW bubble discovered from its $1420 \mathrm{MHz}$ radio continuum (left) and HIRES $60 \mu \mathrm{m}$ (right) emission. The extended radio emission has a thermal spectrum and the central IR source (without a measurable radio counterpart) has a spectral index $\alpha$ (with $S_{\nu} \propto \nu^{-\alpha}$ ) of -0.64 between $60 \mu \mathrm{m}$ and $100 \mu \mathrm{m}$. 

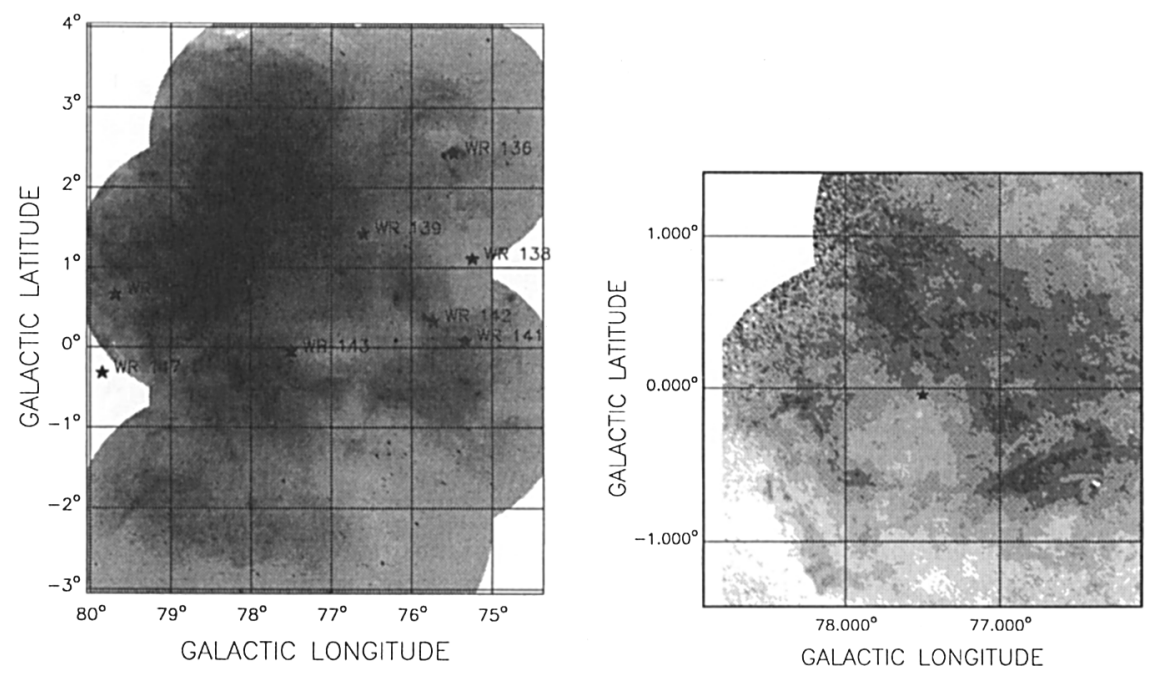

Figure 2. Left: a typical $1420 \mathrm{MHz}$ radio-continuum mosaic formed by adding eight adjacent individual $D R A O$ fields covering a $6^{\circ} \times 7^{\circ}$ area $(l \times b)$ in the Cygnus region of the Galaxy (WR 143 is near the center). Right: the neutral hydrogen emission (centered at $v_{\mathrm{lsr}} \approx-18 \mathrm{~km} \mathrm{~s}^{-1}$ and covering a velocity range of $8 \mathrm{~km} \mathrm{~s}^{-1}$ ) in the vicinity of the star WR 143 (asterisk) showing a possible $\mathrm{H}$ I cavity created by the star.

objects (most notably supernova remnants) is achieved by a determination of the radio spectral index (e.g., Pineault 1998; Pineault \& Terebey 1997). As an illustration of the potential of the project, Figure 1 shows the $1420 \mathrm{MHz}$ radio continuum and HIRES (Fowler \& Aumann 1994) $60 \mu \mathrm{m}$ maps of a new SW bubble. Figure 2 shows a 'typical' CGPS $1420 \mathrm{MHz}$ radio-continuum mosaic (left) and (right) the $\mathrm{H}$ I emission (Pineault \& Cazzolato 1998, in preparation) around the star WR 143. The latter suggests the presence of an H I cavity coincident (although severely offset) with the WR star. The measured LSR-velocity is, however, not consistent with the velocity inferred from the stellar distance (through a galactic rotation curve) and may imply significant non-circular motion.

\section{References}

Fowler, J.W., Aumann, H.H. 1994, in: S. Terebey \& J. Mazzarella (eds.), Science with High-Resolution Far-Infrared Data, JPL Pub. 94-5, 1

Normandeau, M., Taylor, A.R., Dewdney, P.E. 1996, Nature 380, 687

Pineault, S. 1998, AJ 115, 2483

Pineault, S., Terebey, S. 1997, AJ 113, 433 